\title{
Multipactor Effect Analysis and Design Rules for Wedge-Shaped Hollow Waveguides
}

\author{
Jaime Hueso, Carlos Vicente, Benito Gimeno, Member, IEEE, Vicente E. Boria, Senior Member, IEEE, \\ Stephan Marini, Member, IEEE, and Máriam Taroncher, Student Member, IEEE
}

\begin{abstract}
A numerical model for predicting the multipactor breakdown effect in wedge-shaped hollow waveguides is presented in this paper. The computation of electromagnetic fields is based on the boundary integral-resonant mode expansion method, which provides the modal chart of hollow waveguides with any arbitrary cross section. The advantage of using wedge-shaped waveguides with respect to conventional rectangular ones is the deviation of the resonant paths of the electrons toward regions with lower voltages, thus reducing the probability of multipactor threshold for certain input power. To validate this method, our results have been compared with simulations from previous theoretical studies. Once the simulation tool is validated, it is used to predict the multipactor threshold of wedge-shaped waveguides with different symmetric inclination angles of their horizontal plates. Finally, susceptibility curves as the ones already available for rectangular waveguides are presented. These charts are useful for designing innovative waveguide geometries with improved multipactor-free working power ranges.
\end{abstract}

Index Terms-High-power phenomenon, multipactor (MP), radio frequency (RF) breakdown threshold, susceptibility curves, wedge-shaped waveguide.

\section{INTRODUCTION}

$\mathbf{M}$ ULTIPACTOR (MP) is a discharge phenomenon that occurs in RF components operating under vacuum condition [1]. When the free electrons synchronize with the RF field oscillations in the device, an avalanche reaction of the electron population may occur and lead to one or several discharges. These discharges can increase the return losses of the component as well as its temperature. Sustained MP discharges cause outgassing of the walls and even ionization breakdown, which may physically damage the structure and completely disrupt its performance [2].

Manuscript received May 14, 2010; revised August 30, 2010; accepted September 2, 2010. Date of publication October 14, 2010; date of current version November 19, 2010. This work was supported by the Ministerio de Ciencia e Innovación, Spain, under Research Project TEC2007-67630-C0301/TCM. The review of this paper was arranged by Editor W. L. Menninger.

J. Hueso is with the Microwave and Radar Institute, German Aerospace Center (DLR), 82230 Wessling, Germany (e-mail: jaime.hueso@ dlr.de).

C. Vicente is with the Aurora Software and Testing S.L., Universidad Politécnica de Valencia, 46022 Valencia, Spain.

B. Gimeno is with the Departamento de Física Aplicada, Instituto de Ciencia de los Materiales-Universidad de Valencia, 46100 Burjassot, Spain.

V. E. Boria and M. Taroncher are with the Departamento de Comunicaciones, iTEAM, Universidad Politécnica de Valencia, 46022 Valencia, Spain.

$\mathrm{S}$. Marini is with the Departamento de Física, Ingeniería de Sistemas y Teoría de la Señal, Universidad de Alicante, 03080 Alicante, Spain.

Color versions of one or more of the figures in this paper are available online at http://ieeexplore.ieee.org.

Digital Object Identifier 10.1109/TED.2010.2075931

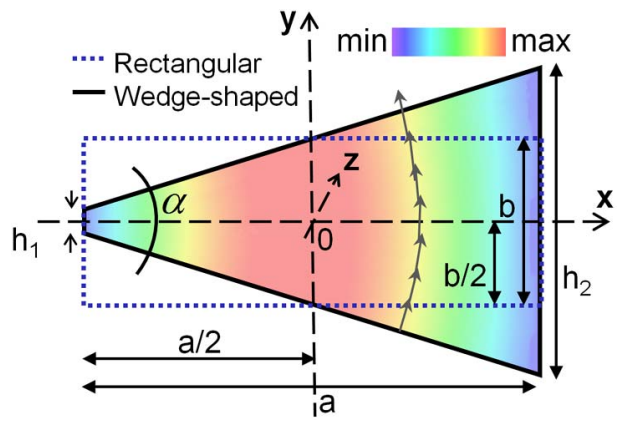

Fig. 1. Cross sections of a wedge-shaped waveguide and of its equivalent rectangular one. Both waveguides have the same width $a$ and average height $b=\left(h_{1}+h_{2}\right) / 2$, resulting in equal cross-section surfaces. Inside the wedgeshaped cross section, the colors indicate the distribution of the transverse electric field of the fundamental mode, similar to the $T E_{10}$ rectangular waveguide fundamental mode. (Gray arrows) Electric field vectors along an arc between top and bottom plates.

This RF breakdown effect has been subject of numerous studies for over 70 years since it may affect a broad range of high-power components and applications, such as passive RF filters for space applications [3], microwave generators and power amplifiers like klystrons and traveling-wave tubes [4], or particle accelerators like cyclotrons [5].

Focusing on microwave hollow waveguides for space applications, it would be desirable to increase their MP-free power range while keeping its size, mass, and electrical behavior. A practical option is to use waveguides with wedge-shaped cross section (CS) instead of the conventional rectangular one. Instead of having parallel top and bottom walls, the wedged CS is achieved by introducing a symmetric inclination angle $\alpha$ between them, as shown in Fig. 1 .

This idea was originally introduced for particle accelerator systems in [6] and [7], and then, it was successfully applied and tested with a bandpass filter operating in the $\mathrm{X}$-band for space applications [8], [9]. Following the interest toward wedgeshaped waveguides [6]-[9], an extended theoretical study of their MP discharge behavior concluded that these waveguides do also have a trapped electron trajectory (see [10]), which defines a critical initial position where the electrons should be launched for the MP prediction simulations.

The consequent step forward is to quantify the real advantage of using wedge-shaped waveguides and facilitate their application to more complex designs. In order to achieve such an aim, in this paper, we derive MP susceptibility curves for wedge-shaped waveguides, analogous to the ones existing for rectangular and parallel plate geometries [11]. 
For this purpose, an efficient simulation tool has been developed, which follows the 3-D trajectory of an effective electron inside an infinite wedge-shaped hollow waveguide. For each input power step, the electron is launched several times with different initial RF phases. The secondary electron emission coefficients (SEECs) obtained in each wall collision are accumulated in a variable for each launched electron. Unlike [6] and [10], where the wedge-shaped waveguide has circular arcs as sidewalls to facilitate its analysis, this paper considers completely straight parallel sidewalls, which are easier to manufacture and are more realistic. A consequence of this is that the electromagnetic (EM) fields cannot be solved analytically (see [6] and [10]). In order to compute the required EM fields, our software code follows the boundary integral-resonant mode expansion (BI-RME) method (see [12] and [13]), which can provide the complete modal chart of any arbitrarily shaped CS. Another difference with respect to previous publications is the tracking of the electron trajectory in 3-D space, which can allow further studies of nonuniform real waveguide structures.

After this introduction, Section II presents the efficient and accurate computation of the required EM fields. Next, Section III describes the main features of the developed software tool for predicting MP, including some validation results. Then, in Section IV, a parametric study for the identification of the optimal inclination angle between the top and bottom plates of a wedge-shaped hollow waveguide is performed. For the first time to the authors' knowledge, susceptibility maps for wedgeshape waveguides are presented, namely, in Section V. Finally, some conclusions and future research areas are summarized in Section VI.

\section{Accurate Computation OF EM Fields}

As it is detailed in Section III-A, in order to calculate the trajectories of the electron within the wedge-shaped waveguide, the computation of its EM fields is needed. The wedged geometries considered in this paper are similar to rectangular waveguides, but their top and bottom walls are symmetrically inclined (see Fig. 1). Unlike the annular section waveguides proposed in [6] and [10], its sidewalls are completely straight; hence, its RF fields cannot be calculated analytically.

The fields of the CS geometry under consideration are computed by means of the BI-RME method [13]. The BIRME technique efficiently computes both the modal cutoff frequencies (eigenvalues) and the electric and magnetic field patterns (eigenvectors) of the wedge-shaped waveguide as a solution of two generalized eigenvalue problems related with the TE and TM modes of the reference rectangular waveguide (see [12] and [13]). The normalized transverse electric fields of the arbitrary waveguide modes can be computed as

$$
\vec{e}_{m}^{\mathrm{arb}}=\sum_{i=1}^{\infty}\left[\left\langle\vec{e}_{m}^{\mathrm{arb}} ; \vec{e}_{i}^{\square}\right\rangle \cdot \vec{e}_{i}^{\square}\right]
$$

where $m=1$ for the fundamental mode, which is the one with the lowest cutoff frequency, and $\square$ symbolizes the reference standard rectangular waveguide. The term $\langle\cdot ; \cdot\rangle$ is the coupling integral in the wedged CS between the arbitrary waveguide mode and a certain one of the set of rectangular waveguide modes, and is calculated as follows:

$$
\left\langle\vec{e}_{m} \vec{e}_{i}\right\rangle=\int_{\mathrm{CS}} \vec{e}_{m} \cdot \vec{e}_{i} d S .
$$

These coupling integrals are provided by the BI-RME method [13], whereas the modes of the rectangular waveguide have a well-known analytical form [12].

However, the direct evaluation of the series in (1) with sufficient accuracy for every iteration step of the electron trajectory algorithm would have an extreme computational cost. In order to increase the efficiency of our numerical code, we just compute the normalized EM fields in a grid of the wedged CS, which is then interpolated in order to retrieve the actual fields in the current position of the electron.

Since waveguides are usually operated at frequencies where only the fundamental mode $(m=1)$ is propagating, in MP prediction simulations, this will be the one chosen to interact with the electrons. In conventional rectangular waveguides, the fundamental mode is a $T E_{10}$ mode. Analogously, wedgeshaped waveguides with small inclination angles, like the one in Fig. 1, have $T E_{10}$-like electric field patterns for their fundamental mode and also similar cutoff frequencies than the ones of rectangular waveguides with the same width. With increasing inclination angles $\left(\alpha>30^{\circ}\right)$, the field patterns have a stronger deformation, and the differences between the cutoff frequencies of the first and second propagating modes decrease. Section IV gives more detailed information on this topic, studying the waveguide properties in terms of the inclination angle between the plates.

\section{MP PREDICTION TOOL}

\section{A. Electron Dynamics}

It is assumed that the electron dynamics are governed by the following Lorentz force equation:

$$
\vec{F}_{L}=q(\vec{E}+\vec{v} \times \vec{B})=\frac{d \vec{p}}{d t}
$$

where $q=-e$ is the electron charge, $\vec{E}$ and $\vec{B}$ are, respectively, the electric and magnetic fields interacting with the electron, and $\vec{v}$ is the velocity vector of the electron. The linear momentum is defined as $\vec{p}=m_{0} \gamma \vec{v}$, where $m_{0}$ is the electron mass at rest, $\gamma=1 / \sqrt{\left(1-(v / c)^{2}\right)}$ is the relativistic factor, with $v$ being the magnitude of the velocity vector, $c=1 / \sqrt{\left(\mu_{0} \epsilon_{0}\right)}$ is the free-space velocity (where $\mu_{0}$ is the free-space magnetic permeability, and $\epsilon_{0}$ is the free-space electric permittivity), and $t$ is the time.

The differential equation in (3) is solved by means of the velocity Verlet algorithm (see [14] and [15]). As it is shown in [16], this ensures accuracy and reasonable numerical efficiency provided that enough time steps (750 steps per RF cycle in this paper) are chosen. Although the relativistic component of this equation can be discarded for the typical power ranges of most space waveguide devices, it should be considered for plasma 

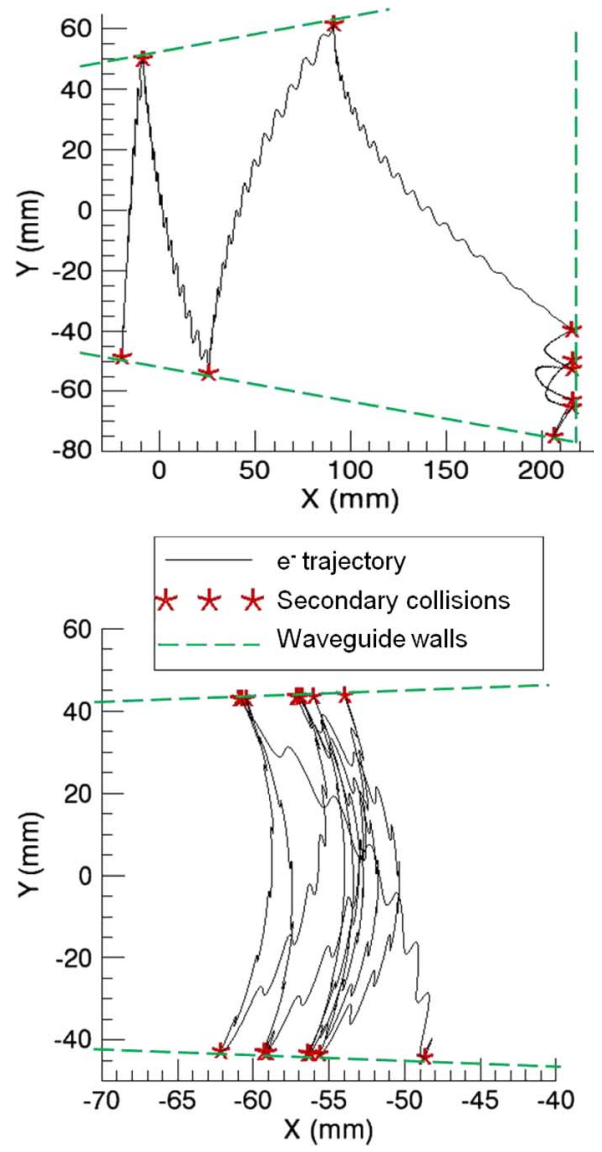

Fig. 2. Two simulations of the trajectory of an electron in the same wedgeshaped cross-section waveguide [6] and its collisions against the walls. The $X Y$-plane is perpendicular to the propagation direction. (Top) Electron is deviated to a sidewall. (Bottom) Electron is in a trapped trajectory.

physics applications due to the extreme speeds reached by the electrons at the working power ranges.

Following this algorithm, we have computed the trajectories of two electrons inside a wedge-shaped waveguide similar to the one considered in [6] and [10], which are plotted in Fig. 2.

\section{B. Algorithm for MP Prediction}

In order to study the MP phenomena in waveguides with arbitrary CS, we have followed a traditional effective electron model [17], but modified according to [18]. Essentially, we have tracked the trajectories of $M$ effective electrons, each one launched at equidistant phases within the RF cycle of the input signal, and then, we have analyzed the generation of secondary electrons in the wall collisions.

The electrons are launched from a common position in the waveguide walls with a fixed initial velocity vector. The trajectory of each effective electron is found by solving numerically its equation of motion (3), detecting, in this way, the collisions with the metallic walls of the waveguides (see the simulation example shown in Fig. 2).

When a collision happens, an electron can be elastically reflected or can produce true secondary ones. In the latter case, instead of tracking all the new emitted electrons, the SEEC $\delta$ is computed, which describes the average number of secondary electrons emitted by this incident particle [19]-[21]. Its value depends on the kinetics of the impact and the electric properties of the metallic surface. The effective electron is reemitted from the impact location with a velocity given by a Gaussian model distribution (corresponding to a Maxwell-Boltzmann energy distribution) with configurable mean and standard deviation depending on the material of the walls. The launching angle of such an electron is obtained using the cosine law distribution [22]. The tracking of the electron finishes when one of the following conditions is fulfilled: maximal RF signal cycles, maximum number of collisions, minimum SEEC in one collision, or minimum accumulated SEEC.

The accumulated collision coefficient for each effective electron is called the multiplicity function (formulated in [17]) and is calculated as follows:

$$
e_{n}^{(j)}=\prod_{i=1}^{n} \delta_{i}^{(j)}
$$

where $j$ denotes the effective electron index, $n$ is the total number of impacts (usually 20), $i$ is the index for each impact, and $\delta_{i}^{(j)}$ is the SEEC calculated for each impact.

For each power step, the multiplicity factors from the $M$ launched electrons can be added together to compute the "enhanced counter function" [17] or the secondary sum. When this function has values higher than $M$, it indicates a statistical electron growth and, therefore, a risk of MP. The lowest power value at which the RF MP breakdown is likely to happen is known as the MP power threshold of the waveguide. The corresponding voltage value at this power threshold is known as the MP voltage breakdown threshold $V_{\text {th }}$.

Typically, there are ranges of input power with MP risk, called MP "windows," interleaved with inactive power ranges from an MP point of view. These windows are the result of MP phenomena of different order [1], [3].

\section{Prediction of the Launch Position of the Initial Electron}

In MP prediction simulations, electrons should be ideally launched into trapped trajectories, since they provide stable resonant paths and may result in the highest breakdown probability. In rectangular waveguides, a trapped electron trajectory exists in the center of the CS (i.e., $x=0$ in Fig. 1). Electrons launched at such point in the $y$ direction do not drift toward the sidewalls [23], as long as the rebounds in the collisions are specular (as it is assumed in the simulations in [6]). This can be simply deduced from (3): The electric field of the $T E_{10}$ mode has no component in the $x$ direction, nor has the cross product of $\vec{v} \times \vec{B}$, since the magnetic field has no $z$ component at the $x=0$ plane. Therefore, the electron will remain in this trapped trajectory, where, in addition, the electric field has a maximum, which constitutes a worst case for MP breakdown risk. Even considering a spread in the launch angle after a collision, like in [10], [23], and in this paper, the electrons statistically tend to stay in this trapped electron trajectory.

In [10], it is theoretically demonstrated that annular section waveguides, which are similar to the wedge-shaped waveguides object of this paper (see Fig. 3), also contain trapped 


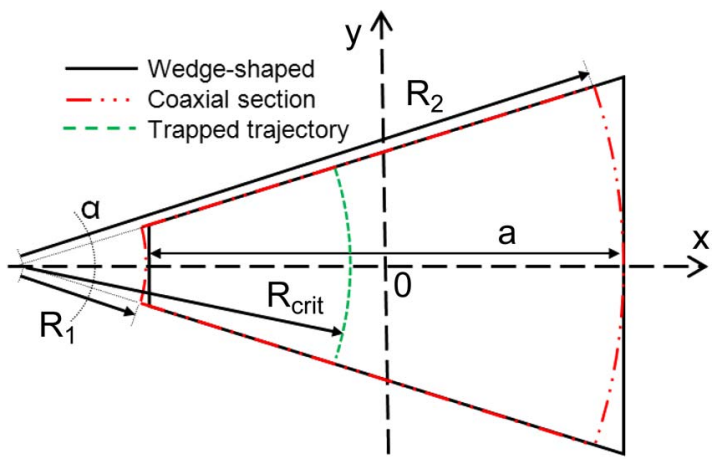

Fig. 3. Definition of the dimensions of an annular section waveguide and its corresponding wedge-shaped waveguide (convention in this paper). $R_{1}$ and $R_{2}$ are the internal and external radii of the annular section, and $R_{\text {crit }}$ is a critical radius with a trapped electron trajectory.

trajectories. In [10], it is proposed to estimate such location as the solution to the following:

$$
\frac{d A\left(R_{0}\right)}{d R_{0}}=2 \frac{A\left(R_{0}\right)}{R_{0}} \cos \phi_{0}^{2}
$$

where $A(r)$ is the vector potential of the fundamental mode of the EM field in annular section waveguides, $\phi_{0}$ is the phase of the RF signal at which the electron starts to interact, and $R_{0}$ is the radius of the trajectory within the waveguide. Equation (5) assumes a zero initial velocity of the launched electron and results in a set of trapped, although unstable, trajectories. This is due to the fact that the solution of the equation depends on $\phi_{0}$, opposed to the rectangular waveguide, where the trapped electron trajectory is independent from this parameter and, therefore, more stable.

Due to the fact that the electric fields in the wedge-shaped waveguides are expressed as summation of weighted rectangular waveguide modes, the derivation of a critical initial position after solving a formula analogous to (5) would be extremely complicated. Instead, each wedge-shaped waveguide is approximated by an equivalent annular section geometry, and then, the same procedure based on solving (5) can be directly followed. The correspondence wedge-to-annular section waveguide is defined as shown in Fig. 3. The approximation is assumed to be valid for values of $\alpha<90^{\circ}$, which is the case for all the geometries under study in this paper.

The strategy for the MP simulation is to launch the initial electrons from the bottom wall of the wedge-shaped waveguide into one of those critical radii $R_{\text {crit }}$ by setting an initial velocity vector tangential to them. The corresponding critical launching locations in the $X Y$ plane can be easily computed as follows:

$$
\begin{aligned}
& x_{\text {crit }}=R_{\text {crit }} \cdot \cos \frac{\alpha}{2}-\frac{R_{1}+R_{2}}{2} \\
& y_{\text {crit }}=-\frac{h_{1}+h_{2}}{4}+\frac{h_{1}-h_{2}}{2 \cdot a} \cdot x_{\text {crit }}
\end{aligned}
$$

where $R_{1}$ and $R_{2}$ are defined in Fig. 3 , and $h_{1}$ and $h_{2}$ are defined in Fig. 1.

In [10], an $R_{\text {crit }}=320.7 \mathrm{~mm}$ was chosen for a wedgeshaped waveguide with dimensions $R_{1}=216 \mathrm{~mm}, R_{2}=$ $648 \mathrm{~mm}, \alpha=0.23 \mathrm{rad}$, and $f=500 \mathrm{MHz}$, when $\phi_{0}=0$.

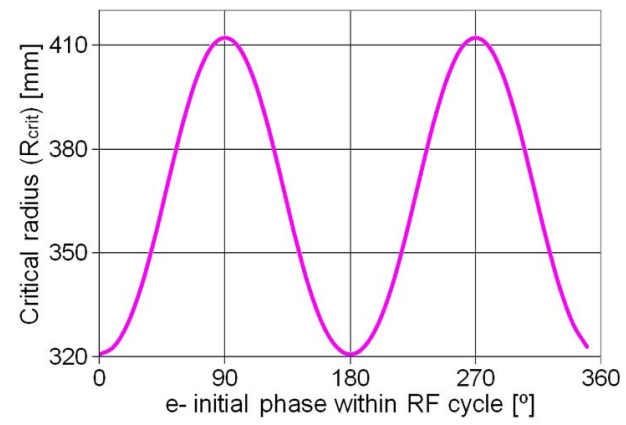

Fig. 4. Launch radii with trapped electron trajectories of the annular section waveguide with dimensions $R_{1}=216 \mathrm{~mm}, R_{2}=648 \mathrm{~mm}$, and $\alpha=0.23 \mathrm{rad}$ [10], depending on the initial launch phase of the electron within the RF signal cycle. The initial velocity of the electron is assumed to be zero.

However, (5) provides a whole range of critical radii, from 320.7 to $412.1 \mathrm{~mm}$, for the different initial phases, as shown in Fig. 4. It has to be noted that the derivation of (5) is done assuming a zero initial velocity of the electron, which is not the case in our simulations. A nonzero velocity complicates the calculation of the stable trajectory of the electrons and does not lead to such a simple closed expression as (5). Hence, it should only be interpreted as a first approximation.

Nevertheless, it is not likely that all trapped trajectories/critical radii have the same impact on the MP behavior of the geometry. The influence of other issues, like the voltage distribution along the waveguide $\mathrm{CS}$, has been considered for establishing additional criteria to identify an eventual "optimal" radius (the highest likelihood of MP breakdown) within the predicted critical range. The concept of "voltage" in wedgeshaped waveguides is not obvious. In this paper, we define it as the line integral of the electric field in a waveguide arc (8), thus depending on the radius where it is calculated, i.e.,

$$
V(R)=\int_{\operatorname{arc}} \vec{E} \cdot \overrightarrow{d l}
$$

This is done so, since the potential trapped electrons will tend to follow the electric field vectors, which, in the wedgeshaped waveguide, approximately describe an arc geometry (see Fig. 1). The integral is calculated numerically with the Simpson method [24].

If the voltage is calculated for all radii of the wedge-shaped waveguide proposed in [10], a curve like the one shown in Fig. 5 is obtained. By overlapping the voltage (solid line) with the critical radii range (shadow region with dashed bounds) shown in Fig. 4, we can observe the relations between them.

Since the MP behavior of a structure is closely linked with the voltage, we can make the assumption that, among the critical radii, the optimal one for MP prediction should be the one with the highest voltage and, therefore, the one which crosses the $x$-axis at $x=-19.9 \mathrm{~mm}$ in Fig. 5. This is the limit of the trapped trajectory region closest to the broader sidewall. Additionally, the fact that the maximum voltage is located outside the trapped trajectory region evidences the effectiveness of wedge-shaped waveguides in increasing the MP threshold. This issue contrasts with rectangular waveguides, where the 


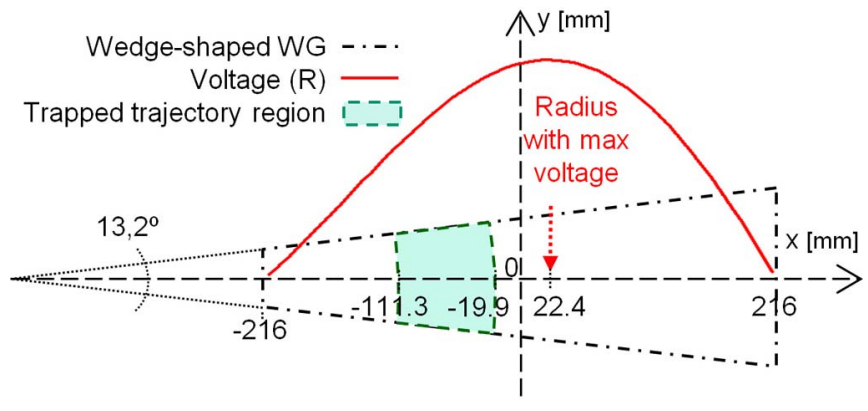

Fig. 5. Cross section of the wedge-shaped waveguide of [10], including the region with trapped electron radii and the voltage amplitude distribution. The qualitative (unitless) distribution of the voltage for the whole radii range. Radius with maximum voltage $R_{\max V}=454.4 \mathrm{~mm}\left(x_{\max V}=22.4 \mathrm{~mm}\right.$ at $\left.y=0\right)$.

voltage maximum is always in the same location as the trapped trajectory.

The methods for the prediction of the launch position of the initial electron proposed in this section have to be demonstrated by means of appropriate simulations, as follows in Section III-D. The additional influence of the voltage in the choice of the optimal trapped trajectory and in the overall MP performance will be verified in Section IV.

\section{Numerical Validation}

In order to validate our software tool, its results are first compared with the ones from [6] and [10]. Our simulations try to reproduce the same conditions, waveguide dimensions, and material properties. Since the power range in all cases is relatively high, the relativistic effect is considered when calculating the electron motion.

Two structures proposed in [6] were chosen for the first comparison, both excited at $f=500 \mathrm{MHz}$ : on one hand, a rectangular waveguide with dimensions $a=432 \mathrm{~mm}$ and $b=$ $102 \mathrm{~mm}$, and, on the other hand, an annular section waveguide with dimensions $R_{1}=216 \mathrm{~mm}, R_{2}=648 \mathrm{~mm}$, and $\alpha=13.2^{\circ}$. For our convenience, the annular section was converted into an equivalent wedge-shaped waveguide with the following characteristics: $a=432 \mathrm{~mm}, h_{1}=49.9841 \mathrm{~mm}, h_{2}=149.952 \mathrm{~mm}$, and $\alpha=13.2^{\circ}$. It has to be noted that the widths of the rectangular and wedge-shaped waveguides are identical, which allows similar simulation conditions and a comparison between both results. The material of the waveguide walls is niobium, and its characteristic SEEC curve can be expressed as follows (see [25]):

$$
\delta(u)=\delta_{m} \frac{1-e^{-A\left(\frac{u}{u_{m}}\right)^{B}}}{C\left(\frac{u}{u_{m}}\right)^{D}}
$$

where $u$ is the energy of the impacting electron (in electronvolts), $\delta_{m}=1.6$ is the maximum SEEC corresponding to a maximum impact energy $u_{m}=200 \mathrm{eV}$, and $A=1.55, B=$ $0.9, C=0.79$, and $D=0.35$ are constants characteristic of this material. The initial electrons and all secondaries are launched with energy of $2 \mathrm{eV}$ normal to the impacting surface.

In Fig. 6, the results of our code (dotted lines) are overlaid with the reference curves in [6] (solid lines). The number of
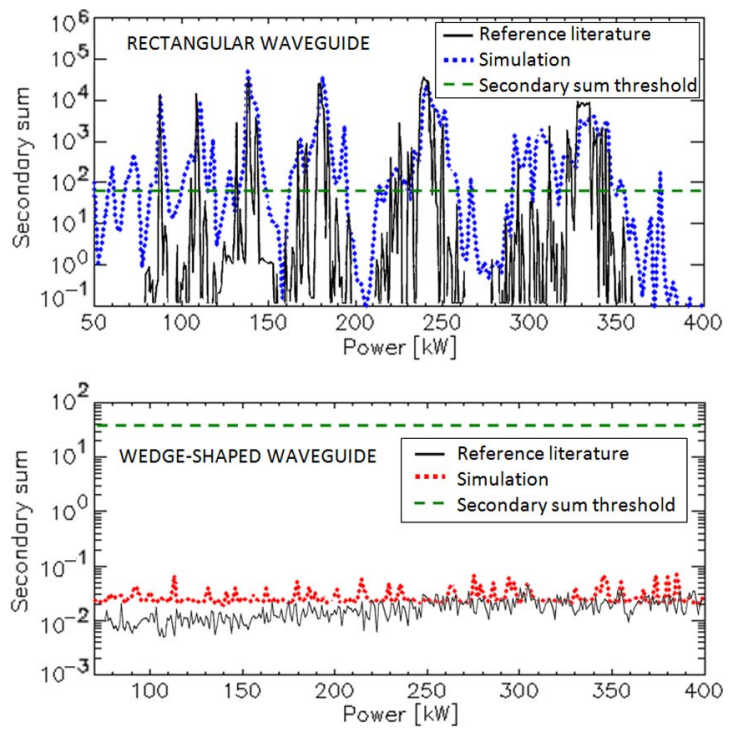

Fig. 6. Comparison of our simulation results (dotted line) with a reference from the literature [6]. (Top) Rectangular waveguide with dimensions $a=$ $432 \mathrm{~mm}, b=102 \mathrm{~mm}$, and the launch position of the initial electron at $x=0$. (Bottom) Wedge-shaped waveguide with dimensions $a=432 \mathrm{~mm}$, $h_{1}=49.9841 \mathrm{~mm}, h_{2}=149.952 \mathrm{~mm}, \alpha=13.2^{\circ}$, and the launch radius at $R=290 \mathrm{~mm}$. In both cases, $M=42$ is the number of launched initial electrons and also the secondary sum threshold value. Twenty maximum wall impacts; 1000 maximum RF cycles; minimum impact energy $0.1 \mathrm{eV}$; minimum accumulated SEEC $10^{-3}$.

launched electrons is $M=42$; hence, this is the secondary sum (enhanced counter function) value that indicates the threshold of the MP risk region.

The top plot of Fig. 6 contains the results of the rectangular waveguide, with some high-risk MP power regions. Both curves show a very good agreement in the shape and the location of these MP windows. The wedge-shaped waveguide results are shown in the bottom plot of Fig. 6. Here, both simulation and reference curves confirm that no MP risk is expected in this case. To stay in the safe side, this simulation was repeated with different initial electron launching positions around the one chosen in [6], i.e., $290 \mathrm{~mm}$, coming to the same conclusion of no MP risk.

In [10], a very similar geometry to the annular section considered in [6] is studied. However, there is a slight modification in the dimensions of the corresponding rectangular waveguide, namely, $a=414.9 \mathrm{~mm}$ and $b=103.6 \mathrm{~mm}$, in order to maintain the same cutoff frequency of the fundamental mode as the one of the annular section. Instead of niobium, the material of the waveguide walls is a silver type with the following characteristic SEEC curve (see [10]):

$$
\delta=\delta_{m}\left[\epsilon \cdot e^{1-\epsilon}\right]^{v(\epsilon)}
$$

where $\delta_{m}=2.22, \epsilon=u / u_{m}, u_{m}=519 \mathrm{eV}, v(\epsilon)=0.62$ if $\epsilon<1$, and $v(\epsilon)=0.25$ if $\epsilon>1$. Furthermore, a spread of the emission velocity and the emission angle after a collision is considered [10] since this is a more reliable method than considering a fix rebound of [6] for detecting an MP risk in wedge-shaped waveguides. The average emission energy is $2.92 \mathrm{eV}$, with a spread that follows a 2-D Maxwellian function (emission energy and angle). The initial electrons are still 

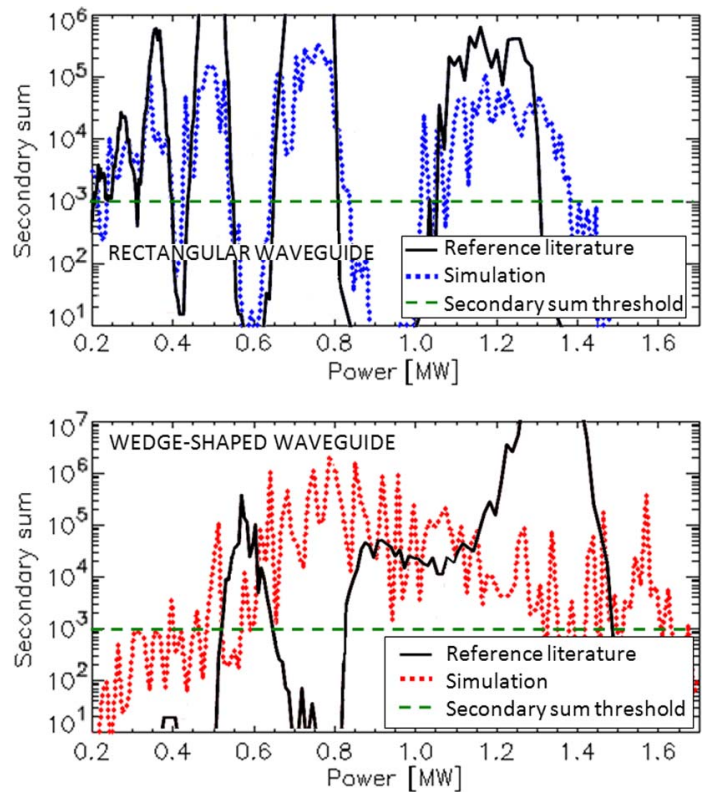

Fig. 7. Comparison of our simulation results (dotted line) with a reference from the literature [10]. (Top) Rectangular waveguide with dimensions $a=$ $414.9 \mathrm{~mm}, b=103.6 \mathrm{~mm}$, and the launch position of the initial electron at $x=0$. (Bottom) Wedge-shaped waveguide with dimensions $a=432 \mathrm{~mm}$, $h_{1}=49.9 \mathrm{~mm}, h_{2}=149.7 \mathrm{~mm}, \alpha=0.23 \mathrm{rad}$, and the launch radius at $R=$ $390.1 \mathrm{~mm}$ (differs from the $R=320.7 \mathrm{~mm}$ in [10]). In both cases, $M=1000$ is the number of initial electrons launched at equidistant RF cycle phases and also the secondary sum threshold value. Twenty maximum wall impacts; 1000 maximum RF cycles; minimum impact energy $0.1 \mathrm{eV}$; minimum accumulated SEEC $10^{-3}$

launched with a velocity vector normal to the wall. The results for both waveguides (the rectangular and wedge-shaped ones) are included in Fig. 7. Although an almost perfect match can be observed in the rectangular waveguide curves in the top of Fig. 7, the wedge-shaped waveguide simulation in the bottom shows a relatively different shape than the reference curve of $[10]$. However, the lower and upper MP power limits $(\approx 0.5$ and $\approx 1.6 \mathrm{MW}$, respectively) fit reasonably well. Next, we explain the reasons for this discrepancy and the implications of these simulation results.

According to Section III-C, the optimal electron initial position for the wedge-shaped waveguide MP simulation should lie around the largest radius within the trapped range $\left(R_{\text {crit }} \approx\right.$ $400 \mathrm{~mm}$ ) since the voltage is higher there (see Figs. 4 and 5). This differs from the strategy of [10], where the simulation was done at $320.7 \mathrm{~mm}$, which is the lowest radius of the trapped range. Looking back at the same simulation in [6], an even lower electron launch radius of $290 \mathrm{~mm}$ is used.

In order to verify the best approach, a series of simulations were performed with a sweep along the full range of initial electron positions. With a launching position corresponding to the radius considered in [10] (320.7 mm), no secondary sum value exceeding the threshold was found along the whole defined input power range. In fact, only the simulations with initial radii between 354 and $415 \mathrm{~mm}$ show MP risks for at least one of the input power steps. Nevertheless, this proves the existence of a range of trapped electron trajectories.

Within this initial radius range with MP risks and for each power step, a sweep over all phases of the initial electron, which is the instant when the electron starts to interact with the EM fields, was performed. The phase of the initial electron, at which the MP resonances were stronger, was almost the same for all launch positions and power steps. This seems to contradict the theory expressed in [10] (see also Fig. 4), which states that, for each phase of the initial electron, a different launch radius should contain the trapped trajectory. The fact that in our simulations, as well as in [6] and [10], the initial velocity of the electron is different from zero $(2.92 \mathrm{eV})$, which is needed in order to avoid too long simulation times, disturbs this dependence. As already suggested in Section III-C, probably just the trapped trajectories with the highest voltage will determine the highest MP resonances. The nonzero initial velocity of the electron and the random direction of the electrons after a collision motivate that electrons end up in this more sensitive trapped trajectories, even if originally launched from other radii. Thus, if the initial speed vector remains constant, all simulation iterations will tend to have similar phase values of the initial electron that result in the strongest MP resonances.

The optimal (critical) initial radius has been identified at around $390.1 \mathrm{~mm}$ (the one used for the simulation of the wedgeshape case in Fig. 7). There, the amounts of power values with MP risks are the biggest ones, the lowest power threshold is found, and the peaks of the enhanced counter function curve are maximum, which means that the resonant mechanism of the electrons is the strongest there.

These simulation results indicate a good agreement with the prediction of $400 \mathrm{~mm}$ of Section III-C, which combines the information of the trapped electron trajectory range prediction with the voltage calculations. In Section IV and Table I, it will be analyzed if this initial position prediction strategy is generally applicable to any wedge-shaped waveguide with arbitrary inclination angles.

The fact that no MP risk was detected at the initial position of $320.7 \mathrm{~mm}$, which contradicts the result of [10], and the different curve shapes in the bottom of Fig. 7, might be due to several facts. As explained before, we are dealing with "equivalent" but not identical waveguide CSs, i.e., annular section and wedgeshaped ones. In addition, both software codes implement completely different algorithms to calculate the EM fields. Small discrepancies in the distribution of the fundamental mode in the CS might motivate displacements of the critical radii and the voltage distribution. Nevertheless, the agreement between both secondary sum curves is still good, mainly in the location of the limits of the MP risk windows.

\section{Study of the Optimal Inclination Angle}

Here, the optimal inclination angle $\alpha$ between the top and bottom plates of the wedge-shaped waveguide is discussed. The simulation parameters and waveguide material properties are the same ones used in Section III-D for the waveguide examples of [10]. Several wedge-shaped waveguides with inclination angles between $1^{\circ}$ and $60^{\circ}$ have been simulated (all at $f=$ $500 \mathrm{MHz}$ ), and the results are summarized in Table I.

Each row contains the inclination angle and dimensions of the wedge-shaped waveguide, the launch positions of the initial electrons with trapped trajectories, the computed voltage, and 
TABLE I

InCLinAtion ANgLE SWEEP-MUltipactor Prediction

\begin{tabular}{|c|c|c|c|c|c|c|c|c|c|c|}
\hline \multirow{2}{*}{$\alpha\left[^{\circ}\right]$} & \multicolumn{3}{|c|}{ WG dimensions (mm) } & \multicolumn{3}{|c|}{$\begin{array}{l}\text { Launch positions with trapped tra- } \\
\text { jectories; } x[\mathrm{~mm}] \text {,bottom wall }\end{array}$} & \multicolumn{3}{|c|}{ Computed voltage for $P=1 W$} & \multirow{2}{*}{$\begin{array}{l}\text { MP } \\
\text { threshold } \\
\text { (simulated) } \\
{[\mathrm{kW}]}\end{array}$} \\
\hline & $a$ & $h_{1}$ & $h_{2}$ & $\begin{array}{l}\text { Start/end } \\
\text { (predicted) }\end{array}$ & $\begin{array}{l}\text { Start/end } \\
\text { (simulated) }\end{array}$ & $\begin{array}{l}\text { With max } \\
\text { MP (simu- } \\
\text { lated) }\end{array}$ & $\begin{array}{l}\operatorname{Max} \\
{[\mathrm{V}]}\end{array}$ & $\begin{array}{l}\text { Location } \\
\max ; x[\mathrm{~mm}] \\
\text { bottom wall }\end{array}$ & $\begin{array}{l}\text { Value } \\
\text { for max } \\
\text { MP [V] }\end{array}$ & \\
\hline$\overline{0}$ & 414.9 & 103.6 & 103.6 & 0 & $-23 / 23$ & $\overline{0}$ & 23.3 & 0 & 23.3 & 170 \\
\hline 1 & 414.94 & 99.98 & 107.2 & $-7.6 /-1.7$ & $-22.0 / 19.0$ & -4.0 & 23 & 3.8 & 23 & 175 \\
\hline 2 & 415.1 & 96.4 & 110.8 & $-15.2 /-3.4$ & $-26.9 / 13.1$ & -8.9 & 23.1 & 4.7 & 23 & 185 \\
\hline 5 & 416.8 & 85.4 & 121.8 & $-38.2 /-8.5$ & $-35.7 / 1.22$ & -13.8 & 23 & 4 & 22.7 & 230 \\
\hline 10 & 422.7 & 66.6 & 140.6 & $-79.2 /-16.9$ & $-56.0 /-14.2$ & -38.0 & 23 & 12.5 & 21.2 & 240 \\
\hline 13.2 & 432 & 49.9 & 149.7 & $-113.4 /-22.6$ & $-80.3 /-19.7$ & -44.6 & 22.4 & 19.4 & 19.8 & 230 \\
\hline 15 & 435.1 & 46.3 & 160.9 & $-126.5 /-24.6$ & $-110.0 /-16.9$ & -57.0 & 22.9 & 27.6 & 19 & 260 \\
\hline 20 & 460.8 & 22.4 & 184.8 & $-188.5 /-28.1$ & $-136.0 /-18.0$ & -67.0 & 23.1 & 37.7 & 16.6 & 240 \\
\hline 25 & 499.7 & 1 & 222.6 & $-248.3 /-16.6$ & $-150.0 /-8.0$ & -67.0 & 24.7 & 58.1 & 15.5 & 330 \\
\hline 30 & 498.3 & 1 & 268.1 & $-247.8 /-18.9$ & $-144.7 /-14.3$ & -62.6 & 27.1 & 55.6 & 17.6 & 400 \\
\hline 35 & 496.5 & 1 & 314 & $-247.2 /-21.6$ & $-116.3 /-2.0$ & -50.0 & 29.2 & 49.9 & 21.1 & 490 \\
\hline 40 & 493.9 & 1 & 360.6 & $-246.1 /-24.7$ & $-125.2 /-4.0$ & -51.9 & 31.3 & 46.4 & 22.4 & 500 \\
\hline 45 & 490.9 & 1 & 407.8 & $-244.7 /-28.2$ & $-126.5 / 3$ & -34 & 33.2 & 50.6 & 26.8 & 580 \\
\hline 50 & 487.6 & 1 & 455.6 & $-243.2 /-32$ & $-127 / 18$ & -32 & 34.9 & 44.6 & 28.8 & 670 \\
\hline 55 & 483.3 & 1 & 504.1 & $-241.2 /-36.2$ & $-118 / 6$ & -42 & 36.7 & 38.4 & 28.9 & 690 \\
\hline 60 & 478.2 & 1 & 553.2 & $-238.7 /-40.6$ & $-114 /-2$ & -40.7 & 37.7 & 40 & 30.1 & 750 \\
\hline
\end{tabular}

the obtained MP power threshold. The predicted start/end of the launch positions is calculated applying (5) and selecting the extreme limits (see Fig. 4), whereas the simulated start/end values correspond to the range of initial electron launch positions where the MP simulation showed at least one power step with MP risk. The initial launch position with the max MP threshold (higher enhanced counter function values and lower power threshold, and, therefore, the optimal for MP prediction) and the maximum voltage were derived like in Section III-D. The position of the initial electron located in this arc is also included. The voltage value for maximum MP corresponds to the voltage along the arc that contains the simulated launch position with maximum MP. It has to be noted that for the wedgeshaped waveguides with inclination angles between $42^{\circ}$ and $60^{\circ}$, the working frequency of $500 \mathrm{MHz}$ also allows the second mode to propagate. However, in this paper, we only consider the excitation of the waveguide through its fundamental mode.

Several conclusions can be extracted from this table. First, the predicted start/end initial positions of the electrons are similar to the simulated ones. The simulated position ranges are larger than the predicted ones for small inclination angles. This might be due to the random rebound angles after a wall collision, which might push back the electrons to trapped trajectories, even if the initial launch position is outside of them. With increasing inclination angles $\left(\alpha>20^{\circ}\right)$, the predicted start position gets closer to the "narrow" part of the wedge shape, whereas the simulated position does not. The justification is that, although resonant trajectories may theoretically exist close to the "narrow" part, the voltage there is too low to generate detectable resonances that lead to the MP effect. Finally, we can see that the initial position with maximum MP risk is always located close to the simulated end position. We can understand this if we realize that the maximum voltage arc is systematically closer to the "broad" part of the wedge shape, and, therefore, the voltage along the critical initial position range increases with the radius (see also Fig. 5). The MP-enhanced counter function curves do not change much when modifying the initial launch

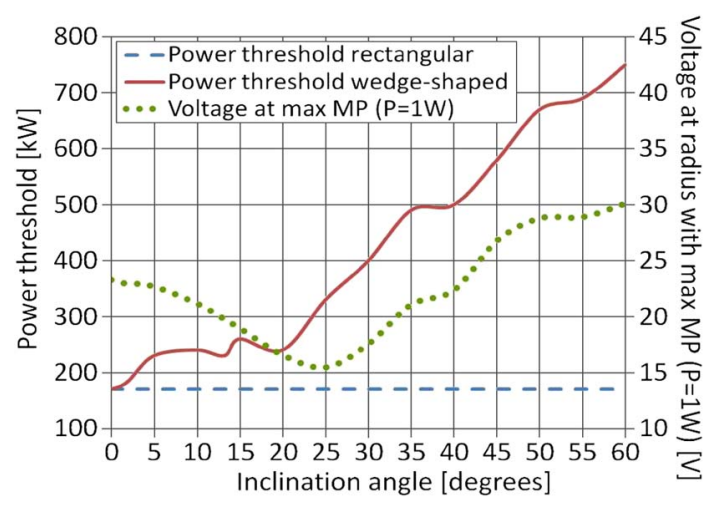

Fig. 8. Multipactor thresholds and voltage values at the MP critical locations for multiple inclination angles.

positions around the optimal, but quickly fade when getting closer to the start/end positions, where the resonances are lost.

These results prove that the approach suggested in Section III-C can be successfully applied to limit the computational effort of the MP predictions for any wedge-shaped waveguide type since only the identified limited range of initial positions has to be simulated.

Concerning the maximum voltage values, they remain very similar to the one of the rectangular waveguide, as long as the average height of the waveguide keeps constant. Once the average height starts to increase $\left(\alpha>25^{\circ}\right)$, the maximum voltage logically increases for the same input power. However, the radius where the strongest MP risk was simulated does not correspond to the position of the maximum voltage. In fact, we can see that the voltage at the position of the strongest MP risk continuously decreases with increasing inclination angles with equal average height.

Fig. 8 shows the voltages and MP thresholds over the different inclination angles. The simulation prediction for the rectangular waveguide shows a constant threshold of $170 \mathrm{~kW}$. The MP thresholds of the wedge-shaped waveguides quickly rise with increasing $\alpha$ and stabilize around $230 \mathrm{~kW}$, around $40 \%$ higher 

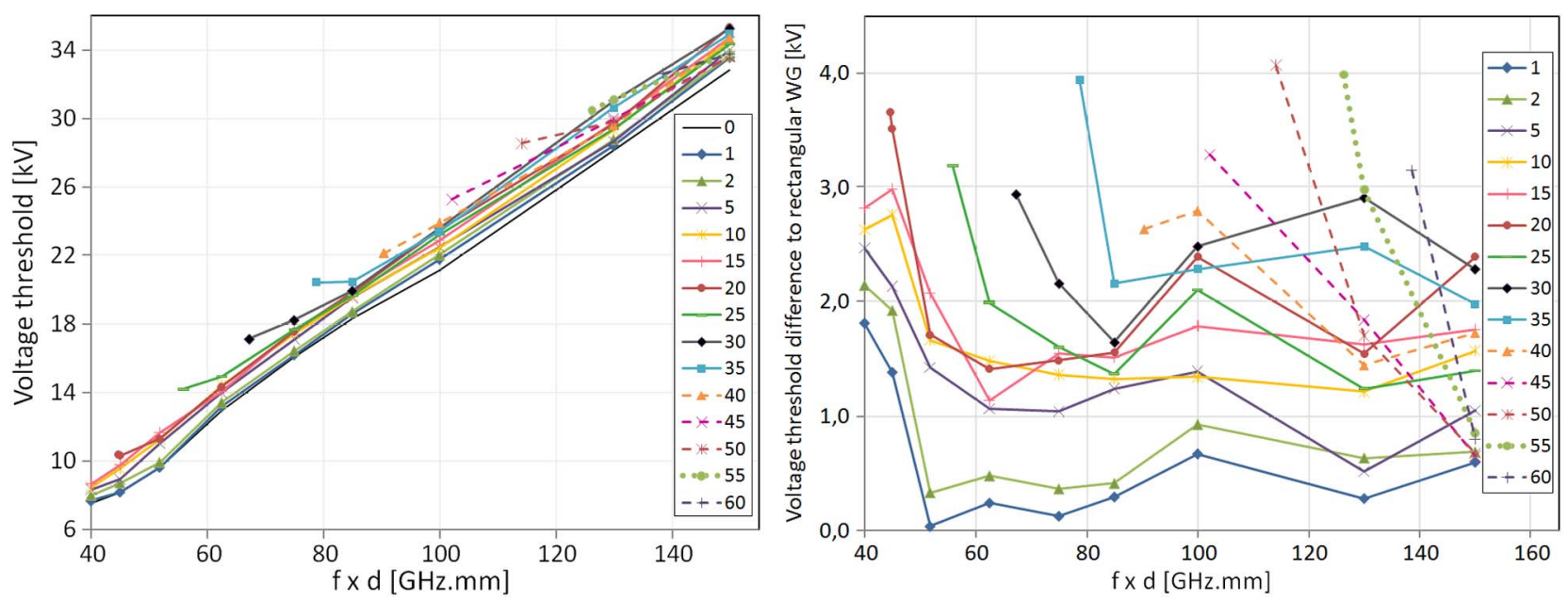

Fig. 9. Susceptibility map for different inclination angles. Thresholds after 20 collisions.

than the one of the equivalent rectangular waveguide threshold. This proves the assumption that wedge-shaped waveguides have a better MP-free power range than rectangular ones. Even small inclination angles present this increment in the MP threshold with respect to the rectangular case.

We can observe that the MP threshold quickly rises with respect to the rectangular waveguide case, even for minimal inclination angles. Between $5^{\circ}$ and $20^{\circ}$, it remains almost constant, and from $25^{\circ}$ on, it rises continuously. However, it has to be noted that this latter rise in the threshold corresponds with the mentioned increase in the mean height of the waveguides.

Concentrating on the voltage curve in Fig. 8, the first remarkable fact is that there is a minimum around $25^{\circ}$. In other simulated waveguides, where inclination angles of $30^{\circ}$ could be designed without increasing the average height, a continuation in the decreasing tendency of the voltages was observed. This suggests using inclination angles around these values or the biggest angle possible below them with equal waveguide mean height. Inclinations between $5^{\circ}$ and $30^{\circ}$ are also a good design compromise, as they have MP threshold values very similar to the optimum case, offering approximately $40 \%$ higher power threshold than the equivalent rectangular waveguide.

\section{SUSCEPTIBILITY MAPS}

\section{A. Definition}

The MP susceptibility maps for a parallel plate model are defined as follows: The abscissa corresponds to the frequency gap product $f \times d$, where $d$ is the distance between the top and bottom plates, and the ordinate represents the voltage or the power of the input signal.

Regions with risk of MP are highlighted, creating the well-known susceptibility curves [11] that indicate the MP phenomena of all possible orders. These curves help in the design of MP-free waveguide structures, usually in rectangular waveguide devices for space applications.

In order to provide a similar tool for the design of wedgeshaped waveguides, the following susceptibility map is proposed: The frequency gap product is obtained by multiplying the working frequency by the mean height of the waveguide.
A different curve will be obtained per inclination angle of the wedge-shaped geometry.

\section{B. Simulated Curves}

By simulating a high number of wedge-shaped waveguides at different frequencies, with different gap sizes and inclination angles, the following susceptibility maps were generated. The curves (see Fig. 9) show the MP voltage threshold $V_{\text {th }}$ of the devices (first voltage, at which an MP risk is detected) and can be compared with the ones of the corresponding rectangular waveguide. The same simulation conditions and waveguide materials of Section IV are used here.

The susceptibility curves for all angles verify the results obtained in the previous sections. The $V_{\text {th }}$ values are always higher than in the rectangular waveguide case, which coincides with the case $\alpha=0^{\circ}$. The improvement in the $V_{\text {th }}$ reaches a factor of 1.35, depending, of course, on the inclination angle. Small angles up to $5^{\circ}$ degrees are fairly close to the rectangular threshold. $V_{\text {th }}$ usually increases with the angle until around $35^{\circ}$, falling slightly again for higher angles. $V_{\text {th }}$ at $30^{\circ}$ seems to be the optimum for many of the cases considered in the simulation range; however, the improvement with respect to the other angles is not dramatic. For the lower $f \times d$ values (being $d$ the mean height), no results are available for large inclination angles. No wedge-shaped geometries with such large angles were possible since the mean height was too low, and the "narrow" side of the CS reached its minimum design limit of $1 \mathrm{~mm}$. When traveling toward higher abscissa values, these inclination angles become gradually feasible, like in the case of $25^{\circ}$ at $f \times d=56$ or $35^{\circ}$ at $f \times d=79$.

\section{CONCLUSION}

In this paper, a numerical model for predicting the MP RF breakdown thresholds in wedge-shaped waveguides has been presented. The tool uses the BI-RME method in order to consider wedge-shaped waveguides with straight sidewalls, which are easier to manufacture than annular section waveguides, but much more complex to analyze. 
The software tool has been verified with simulations available in the literature, and then, it has been used to analyze the MP behavior depending on the inclination angle between top and bottom walls. This allows confirming the advantage of wedge-shaped waveguides with respect to rectangular waveguides in terms of MP breakdown resistance, as they are able to handle typically $40 \%$ higher power.

The improvement of the thresholds with respect to the rectangular waveguide case has to do with the fact that the voltage values in the trapped electron trajectories are lower than the absolute voltage maxima in the CS. This depends on the inclination angle, which should lie between $5^{\circ}$ and $30^{\circ}$.

Finally, susceptibility curves have been derived for this kind of waveguides. This will allow the application of these innovative geometries to more complex microwave devices, like irises, impedance adaptors, low-pass filters, bandpass filters, or multiplexers, which can attract the interest of the telecommunications and space industry or the plasma physics community.

Future work will deal with other wedge-shaped waveguides of interest and will include efficient MP threshold predictions of more complex structures.

\section{ACKNOWLEDGMENT}

The authors would like to thank D. Raboso from ESA/ESTEC and the rest of the Microwave and MillimeterWave Section and the Payload Systems Laboratory for their support to this research activity.

\section{REFERENCES}

[1] J. R. M. Vaughan, "Multipactor," IEEE Trans. Electron Devices, vol. 35, no. 7, pp. 1172-1188, Jul. 1988.

[2] J. Chang, P. Lawless, and T. Yamamoto, "Corona discharge processes," IEEE Trans. Plasma Sci., vol. 19, no. 6, pp. 1152-1166, Dec. 1991.

[3] A. Woode and J. Petit, "Diagnostic investigations into the multipactor effect, susceptibility zone measurements and parameters affecting a discharge," ESA-Working Paper 1556, Nov. 1989, Tech. Rep.

[4] C. Hill and R. Carter, "Investigation of possible multipactor discharge in a Klystron input cavity," in Proc. Vac. Electron. Conf., 2006, pp. 81-82.

[5] M. A. Furman, "The Electron-Cloud Effect in the Arcs of the LHC," CERN, Geneva, Switzerland, CERN-LHC-Project-Rep.-180, May 1998, Tech. Rep.

[6] E. Chojnacki, "Simulation of a multipactor-inhibited waveguide geometry," Phys. Rev. Spec. Top.-Accel. Beams, vol. 3, no. 3, p. 032001 , Mar. 2000

[7] R. L. Geng, H. Padamsee, S. Belomestnykh, P. Goudket, D. M. Dykes, and R. G. Carter, "Suppression of multipacting in rectangular coupler waveguides," Nucl. Instrum. Methods Phys. Res. A, Accel. Spectrom. Detect. Assoc. Equip., vol. 508, no. 3, pp. 227-238, Aug. 2003.

[8] F. Quesada, V. Boria, B. Gimeno, D. Rebenaque, J. Garcia, A. Melcón, J. Hueso, D. Schmitt, D. Raboso, C. Ernst, and I. Carpintero, "Investigation of multipaction phenomena in passive waveguide filters for space applications," in Proc. IEEE MTT-S Int. Microw. Symp. Dig., Jun. 2006, pp. 246-249.

[9] J. Hueso, D. Raboso, and D. Schmitt, "Microwave waveguide filter with non-parallel plates," Patent eSA/PAT/520, Jun. 5, 2009.

[10] V. Semenov, E. Rakova, N. Zharova, D. Anderson, M. Lisak, and J. Puech, "Simulations of the multipactor effect in hollow waveguides with wedge-shaped cross section," IEEE Trans. Plasma Sci., vol. 36, no. 2, pp. 488-493, Apr. 2008.

[11] A. Hatch and H. Williams, "The secondary electron resonance mechanism of low-pressure high-frequency gas breakdown," J. Appl. Phys., vol. 25, no. 4, pp. 417-423, Apr. 1954.

[12] G. Conciauro, M. Guglielmi, and R. Sorrentino, Advanced Modal Analysis: CAD Techniques for Waveguide Components and Filters. Hoboken, NJ: Wiley, 1998.
[13] S. Cogollos, S. Marini, V. E. Boria, P. Soto, A. Vidal, H. Esteban, J. V. Morro, and B. Gimeno, "Efficient modal analysis of arbitrarily shaped waveguides composed of linear, circular, and elliptical arcs using the BI-RME method," IEEE Trans. Microw. Theory Tech., vol. 51, no. 12, pp. 2378-2390, Dec. 2003.

[14] L. Verlet, "Computer 'experiments' on classical fluids. I. Thermodynamical properties of Lennard-Jones molecules," Phys. Rev., vol. 159, no. 1, pp. 98-103, Jul. 1967.

[15] Q. Spriter and M. Walter, "Classical molecular dynamics simulation with the velocity Verlet algorithm at strong external magnetic fields," J. Comput. Phys., vol. 152, no. 1, pp. 102-119, Jun. 1999.

[16] A. Perez, C. Tienda, C. Vicente, S. Anza, J. Gil, B. Gimeno, V. E. Boria, and D. Raboso, "Prediction of multipactor breakdown thresholds in coaxial transmission lines for travelling, standing and mixed waves," IEEE Trans. Plasma Sci., vol. 37, no. 10, pp. 2031-2040, Oct. 2009.

[17] E. Somersalo, P. Ylä-Oijala, D. Porch, and J. Sarvas, "Computational methods for analyzing electron multipacting in RF structures," Particle Accel., vol. 59, pp. 107-141, 1998.

[18] A. Coves, G. Torrregrosa-Penalva, C. Vicente, B. Gimeno, and V. E. Boria, "Multipactor discharges in parallel-plate dielectric-loaded waveguides including space-charge effects," IEEE Trans. Electron Devices, vol. 55, no. 9, pp. 2505-2511, Sep. 2008.

[19] J. R. M. Vaughan, "A new formula for secondary emission yield," IEEE Trans. Electron Devices, vol. 36, no. 9, p. 1963, Sep. 1989.

[20] A. Shih and C. Hot, "Secondary emission properties as a function of the electron incidence angle," IEEE Trans. Electron Devices, vol. 40, no. 4, p. 824, Apr. 1993.

[21] C. Vicente, M. Mattes, D. Wolk, H. Hartnagel, J. Mosig, and D. Raboso, "Multipactor breakdown prediction in rectangular waveguide based components," in Proc. IEEE MTT-S Int. Microw. Symp. Dig., Jun. 2005, pp. 1055-1058.

[22] J. Lara, F. Pérez, M. Alfonseca, L. Galán, I. Montero, E. Román, and D. Raboso, "Multipactor prediction for on-board spacecraft RF equipment with the MEST software tool," IEEE Trans. Plasma Sci., vol. 34, no. 2, pp. 476-484, Apr. 2006.

[23] V. Semenov, E. Rakova, D. Anderson, M. Lisak, and J. Puech, "Multipactor in rectangular waveguides," Phys. Plasmas, vol. 14, no. 3 , p. 033 501, Mar. 2007.

[24] E. Whittaker and G. Robinson, The Trapezoidal and Parabolic Rules: A Treatise on Numerical Mathematics. New York: Dover, 1967.

[25] R. L. Geng and H. Padamsee, "Exploring multipacting characteristics of a rectangular waveguide," in Proc. Particle Accel. Conf., Apr. 1999, vol. 1, pp. $429-431$.

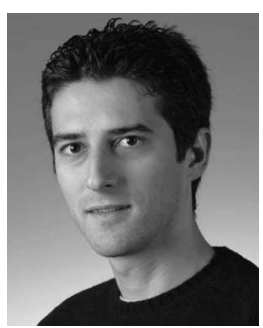

Jaime Hueso received the M.S. degree in telecommunication engineering from the Polytechnic University of Valencia, Valencia, Spain, in 2003.

From 2004 to 2005, he was a Microwave Engineer with the European Space Agency, Noordwijk, The Netherlands, where he researched on microwave filter design, high-power testing, and multipactor effects on spaceborne microwave waveguides. Since 2006, he has been with the German Aerospace Center (DLR), Wessling, Germany, and is currently involved in satellite synthetic aperture radar missions like TerraSAR-X, TanDEM-X, and GMES Sentinel-1.

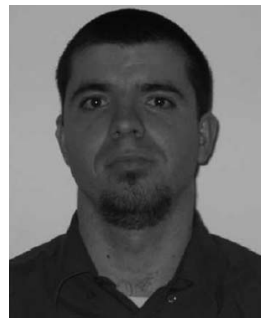

Carlos Vicente was born in Elche, Spain, in 1976. $\mathrm{He}$ received the Dipl. degree in physics from the University of Valencia, Valencia, Spain, in 1999 and the Dr.-Ing degree in engineering from the Technical University of Darmstadt, Darmstadt, Germany, in 2005 .

From 1999 until the beginning of 2001, he was a Research Assistant with the Department of Theoretical Physics, University of Valencia. From 2001 until 2005, he was a Professor Assistant with the Institute of Microwave Engineering, Technical University of Darmstadt. Since 2005, he has been with the Multimedia Applications Group, Technical University of Valencia, Valencia. In 2006, he cofounded the Aurora Software and Testing S. L. devoted to the telecommunications sector. His research concerns passive intermodulation, corona discharge, and multipaction in communications satellite applications. 


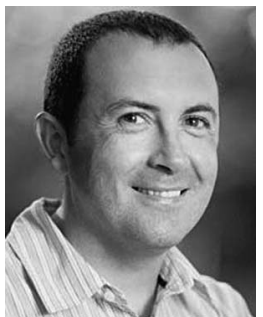

Benito Gimeno (M'01) was born in Valencia, Spain, on January 29, 1964. He received the Licenciado degree in physics and the Ph.D. degree from the Universidad de Valencia, Valencia, in 1987 and 1992, respectively.

From 1987 to 1990 , he was a Fellow with the Universidad de Valencia. Since 1990, he has been an Assistant Professor with the Departamento de Física Aplicada y Electromagnetismo, Universidad de Valencia, where, in 1997, he became an Associate Professor. During 1994 and 1995, he was with the European Space Research and Technology Centre of the European Space Agency as a Research Fellow. In 2003, he obtained a fellowship from the Spanish Government for a short stay (three months) at the Università degli Studi di Pavia, Pavia, Italy, as a Visiting Scientific. His current research interests include the areas of computer-aided techniques for analysis of passive components for space applications, waveguides and cavities including dielectric objects, electromagnetic band-gap structures, frequency selective surfaces, and nonlinear phenomena appearing in power microwave subsystems (multipactor and corona effects).

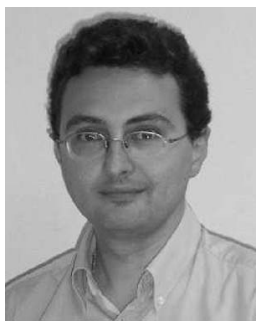

Vicente E. Boria (S'91-A'99-SM'02) was born in Valencia, Spain, on May 18, 1970. He received the "Ingeniero de Telecomunicación" degree (with first-class honors) and the "Doctor Ingeniero de Telecomunicación" degree from the Universidad Politécnica de Valencia, Valencia, in 1993 and 1997, respectively.

In 1993, he joined the "Departamento de Comunicaciones," Universidad Politécnica de Valencia, where, since 2003, he has been a Full Professor. In 1995 and 1996, he held a Spanish Trainee position with the European Space Research and Technology Centre, European Space Agency, Noordwijk, The Netherlands, where he was involved in the area of EM analysis and design of passive waveguide devices. He has authored or coauthored five chapters in technical textbooks, 65 papers in refereed international technical journals, and over 150 papers in international conference proceedings. His current research interests are focused on the analysis and automated design of passive components, left-handed and periodic structures, as well as the simulation and measurement of power effects (multipactor and corona) in passive waveguide systems.

Dr. Boria has been a member of the IEEE Microwave Theory and Techniques Society and the IEEE Antennas and Propagation Society since 1992. He is a member of the editorial boards of the IEEE TRANSACTIONS ON MICROWAVE THEORY AND TECHNIQUeS and the IEEE Microwave and Wireless Components Letters. He is also a member of the technical committees of the IEEE-MTT International Microwave Symposium and the European Microwave Conference. He was the recipient of the 2001 Social Council of the Universidad Politécnica de Valencia First Research Prize for his outstanding activity during the period 1995-2000.

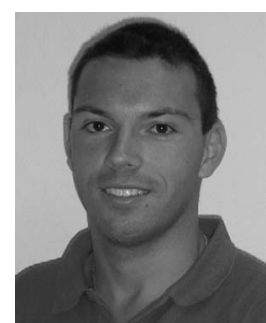

Stephan Marini (M'10) was born in Cagli, Italy, on January 3, 1976. He received the Laurea degree in electronics engineering from the University of Perugia, Perugia, Italy, in 2001 and the Ph.D. degree in telecommunications from the Universidad Politécnica de Valencia, Valencia, Spain, in 2005.

In June 2001, he joined the Departamento de Comunicaciones, Universidad Politécnica de Valencia, Valencia, in the frame of the European Union project "Millimeter-wave and Microwave Components Design Framework for Ground and Space Multimedia Network." Since 2005, he has been a Lecturer with the Departamento de Física, Ingeniería de Sistemas y Teoría de la Señal, Universidad de Alicante, Alicante, Spain. In 2009, he obtained the "José Castillejo" postdoctoral fellowship from the Spanish Government for a short stay (four months) at the LEMA laboratory (École Polytechnique Fédérale de Lausanne, Switzerland). His research interests include numerical methods for the analysis of arbitrary-shaped waveguide, scattering, and periodic structures.

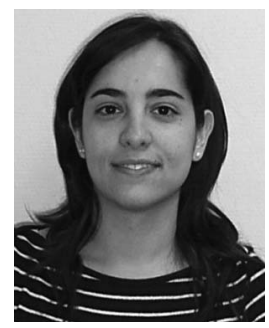

Máriam Taroncher (S'03) was born in Lliria, Valencia, Spain, on October 8, 1979. She received the telecommunications engineering degree in 2003 from the Universidad Politécnica de Valencia (UPV), Valencia, where he is currently working toward the $\mathrm{Ph} . \mathrm{D}$. degree.

From 2002 to 2004, she was a Fellow Researcher with the UPV. Since 2004, she has been a Technical Researcher in charge of the experimental laboratory for high power effects in microwave devices at the Research Institute iTEAM, UPV. In 2006, she was awarded a Trainee position with the European Space Research and Technology Centre, European Space Agency, Noordwijk, The Netherlands, where she worked in the Payload Systems Division Laboratory in the area of multipactor, corona discharge, and passive intermodulation (PIM) effects. Her current research interests include numerical methods for the analysis of waveguide structures and the acceleration of the electromagnetic analysis methods. 